\author{
Aus dem Institut fur Tierzucht und Tierverhalten ${ }^{1}$ Neustadt-Mariensee und dem Institut für Tierernăhrung ${ }^{2}$ der \\ Bundesforschungsanstalt fur Landwirtschaft Braunschweig-Volkenrode \\ ERHARD KALLWEIT', NAHID PARVIZI', FRANC KLOBASA', MARTINA HENNING' \\ und HARTWIG BÖHME ${ }^{2}$
}

\title{
Ebermast mit unterschiedlicher Proteinversorgung
}

\author{
Herrn Professor Dr. Peter Glodek zum 65. Geburtstag gewidmet
}

\begin{abstract}
Summary
Title of the paper: Boar fattening with different protein supply in the diet

In the literature numerous studies are found that proof a superior fattening performance and carcass composition for boars compared to female and castrated pigs. The results in the feeding experiment presented here do not confirm this to the same extend. Even with a high protein and lysine supplementation growth performance of boars was lower than in females. Lean content was determined by FOM grading which might underestimate the boars. Lean to fat ratio (areas of $\mathrm{M}$. long dorsi and back fat) however was significantly different between castrates and all boar groups due to the reduced fat deposition.

Sensoric tests detected significant boar taint in the majority of the samples from male carcasses. Considering the tendency on the market for increasing carcass weights and the risk of boar taint in heavier pigs boar fattening can not be recommended in Germany.
\end{abstract}

Key Words: boar fattening, protein supply, boar taint, meat quality

\section{Zusammenfassung}

Die in der Literatur häufig beschriebene wirtschaftliche Ưberlegenheit der Ebermast konnte an Hand der vorgelegten Ergebnisse nicht im vollen Umfang bestätigt werden. Bei hohen Eiweiß- und Lysingehalten in der Futtermischung war die Wachstumsleistung der Eber nur geringfügig höher als die der Sauen und Börge. Im Magerfleischanteil (FOM) wurden die Eber signifikant von den Sauen übertroffen und unterschieden sich nicht signifikant von den Börgen. Auf Grund der geringeren Fettbildung errechnete sich jedoch ein besseres Fleisch:Fett-Verhältnis für die Eber.

Die sensorische Prüfung ergab bei der Mehrzahl der Eber einen deutlichen Geschlechtsgeruch. Angesichts des Trends zu steigenden Mastendgewichten und des sich daraus ergebenden Risikos einer Geruchsbelastung ist es nicht zu empfehlen, die Ebermast in Deutschland in größerem Umfang durchzufuihren.

Schlüsselwörter: Ebermast, Proteinversorgung, Ebergeruch, Fleischqualität

Die Ebermast hat durch die seit dem 01.01.1993 gültige Richtlinie „Frisches Fleisch (91/497) EWG“ ein in unregelmäßigen Abständen wiederkehrendes Interesse gewonnen. Nach dieser Richtlinie können Schlachtkörper von Ebern im Gewicht von bis zu $80 \mathrm{~kg}$ ohne weitere Untersuchung auf geschlechtspezifischen Geruch in den Verkehr gebracht werden. Zahlreiche Experimente hatten immer wieder bestätigt, daß die anabole Wirkung der Steroidhormone bei Ebern zu einem geringeren Fett- und einem verbesserten Fleischansatz führen. Dies war schließlich auch das Ergebnis einer gemeinsamen Studie von fünf deutschen Forschungsinstituten, die im Jahr 1995 in der Schriftenreihe des Bundesministeriums für Ernährung, Landwirtschaft und Forsten - 
Angewandte Wissenschaft - Heft 449 unter dem Titel „Die Ebermast” veröffentlicht wurden. In dieser Studie wurden die verschiedenen Aspekte der Ebermast von der Mastleistung über die Schlachtkörperzusammensetzung bis hin zu dem Auftreten von Ebergeruch bei verschiedenen Mastendgewichten untersucht und anhand von Literaturquellen ausführlich diskutiert. Es kamen dabei verschiedene Rassenkombinationen zum Einsatz. Die Mast erfolgte bis zu vorgegebenen Mastendgewichten nach einem einheitlichen Fütterungsregime, das dem der Mastleistungsprüfung entspricht. In der hier vorliegenden Untersuchung sollte in Ergänzung zu den bereits dokumentierten Ergebnissen geprüft werden, ob das höhere Fleischbildungsvermögen der Eber lediglich durch eine bessere Verwertung des zugeführten Eiweißes bedingt ist oder ob durch zusätzliche Eiweißgaben eine weitere Steigerung möglich ist. Neben dem Gehalt an verdaulichem Eiweiß wurden auch die zugesetzten Lysinmengen variiert. Die Mast erfolgte im Gegensatz zu der zitierten Studie nicht nach vorgegebenen Mastendgewichten, sondern nach definierten Zeitabschnitten. Durch entsprechende Hormonanalysen sollte der Eintritt der Geschlechtsreife und die sich daraus ergebenden Konsequenzen für den geschlechts-spezifischen Geruch des Fleisch- bzw. Fettgewebes in die Betrachtung einbezogen werden.

\section{Tiermaterial}

Alle Versuchstiere gehörten dem Hannoverschen 3 Rassen-Kreuzungsprogramm (HDP) an (Pi x (DE x DL)). Sie stammten von spezialisierten Ferkelerzeugerbetrieben und wurden durch Mitarbeiter der Landwirtschaftskammer Hannover ausgewählt. Dabei wurde darauf geachtet, daß die Ferkel eines Wurfes gleichmäßig über die Gruppen verteilt wurden. Die Kontrollgruppen bestanden aus 40 Kastraten (I) und 40 weiblichen Tieren (II). Die Gruppen III - VI waren mit jeweils 40 Ebern besetzt.

\section{Fütterung}

Der Energiegehalt sollte für alle Fütterungsgruppen 12,6 ME-MJ/kg betragen. Beim Protein sah die Planung für die Kontrollgruppen und zwei der vier Ebergruppen einen Gehalt von $160 \mathrm{~g} / \mathrm{kg}$ vor, während Lysin in der Kontrollgruppe und zwei Ebergruppen mit $9 \mathrm{~g} / \mathrm{kg}$ enthalten sein sollte; zwei weitere Ebergruppen waren mit $10 \mathrm{~g} / \mathrm{kg}$ vorgesehen. Die Zahlen gehen im einzelnen aus Tabelle 1 hervor. Die Analysewerte zeigen, daß die Planzahlen nicht in jedem Einzelfall genau erreicht wurden. Die Formulierung der Futterzusammensetzung erfolgte in Anlehnung an das Mastleistungsfutter (13 MJ $/ \mathrm{kg}, 16 \%$ Rohprotein, 1\% Lysin) mit den genannten Varianten bei ad lib. Fütterung.

Versuchsbeginn war bei einem Alter der Tiere von ca. 80 Tagen vorgesehen, das Ende der Untersuchung nach 100 Masttagen. Auch hiefür gehen die Soll- und Ist-Werte aus Tabelle 1 hervor.

\section{Methoden}

Die Mast wurde in der LPA Katlenburg durchgeführt, dort wurden den Ebern und Ka- 
straten bei Gewichten von 30 bzw. 60 kg Blutproben aus der Vena jugularis entnommen und auf Testosteron untersucht. Die Schlachtung erfolgte im Versuchsschlachthaus Mariensee bei einem durchschnittlichen Mastalter von ca. 180 Tagen (Tab. 1). Dort wurde an den insgesamt 10 Schlachttagen Schlachtblutproben zur letztmaligen Bestimmung von Testosteron und Progesteron gewonnen. Die Ermittlung von Schlachtkörperwert und Fleischbeschaffenheit fand nach den üblichen LPA-Richtlinien statt. Darüber hinaus wurden im $M$. longissimus dorsi der Wasser- und Fettgehalt mit der Nahinfrarot-Spektroskopie (NIT) bestimmt.

Die Sensorik wurde in Form einer Geruchsprobe bei einem ca. $5 \mathrm{~g}$ schweren Stück Bauch vorgenommen. Dieses Bauchstück wurde in eine Petrischale gelegt und in der Mikrowelle kurz erhitzt; die Tester (nicht speziell geschulte Mitarbeiter des Fleischlabors Mariensee) gaben nach einem kurzen Riecher ihr Urteil ab ( 1 = riecht nicht, 2 = riecht etwas, 3 = riecht stark, 4 = riecht sehr stark).

Weiterhin wurden in Fettproben (Rückenspeck) Skatolgehalte mit Hilfe der HPLC bestimmt (GIBBS et al., 1991).

Die Auswertung wurde anhand einer GLM-Prozedur mit SAS durchgeführt, wobei die sechs Versuchsgruppen auf signifikante Differenzen untersucht wurde. Die Fleischbeschaffenheitswerte wurden schlachttagskorrigiert.

Tabelle 1

Einteilung in Fütterungsgruppen (Soll- und Ist-Werte) (Feeding groups with different protein supply (prèsumed and real contents of energy, lysine and protein))

\begin{tabular}{|c|c|c|c|c|c|}
\hline Gruppe & $\begin{array}{c}\text { I, II } \\
\text { (Kontrollen, } \\
\text { Kastr., weib.) } \\
\text { je } n=40\end{array}$ & $\begin{array}{l}\text { Eber } \\
n=40\end{array}$ & $\begin{array}{l}\text { Eber } \\
n=40\end{array}$ & $\begin{array}{l}\text { Eber } \\
\mathrm{n}=40\end{array}$ & $\begin{array}{l}\text { Eber } \\
\mathrm{n}=40\end{array}$ \\
\hline Ist-Wert & $n=38, n=41$ & $n=39$ & $n=37$ & $n=35$ & $\mathrm{n}=37$ \\
\hline ME-MJ/kg (Soll) & 12,6 & 12,6 & 12,6 & 12,6 & 12,6 \\
\hline Ist-Wert & 13,0 & 13,0 & 12,9 & 13,0 & 12,6 \\
\hline Lysin $\mathrm{g} / \mathrm{kg}$ (Soll) & 9,0 & 9,0 & 9,0 & 10,0 & 10,0 \\
\hline Ist-Wert & 9,4 & 9,4 & 9,2 & 10,2 & 10,2 \\
\hline Protein g/kg (Soll) & 160 & 160 & 180 & 160 & 180 \\
\hline Ist-Wert & 165 & 165 & 175 & 155 & 179 \\
\hline Prüfbeginn & 80,0 & 81,4 & 80,5 & 79,7 & 80,2 \\
\hline Prüfende & 178,0 & 179,2 & 178,2 & 177,4 & 178,2 \\
\hline
\end{tabular}

\section{Ergebnisse}

\section{Mastleistung}

Aus den Daten für die Mastleistungen in Tabelle 2 ist ersichtlich, daß sich die Gruppen im Mastendgewicht bei 180 Tagen Alter nicht wesentlich unterschieden.

Die höchsten Zunahmen erzielten die beiden Ebergruppen V und VI und die Kastraten als Kontrollgruppe, während die beiden Ebergruppen mit dem etwas geringeren Lysin- 
Tabelle 2

Mastverlauf (LSQ-Mittelwerte $(\mu+c)$ und Standardfehler $\left(s_{c}\right)$ der Merkmale der Mastleistung) (Fattening performance (LSQ means $(\mu+\mathrm{c})$ and standard errors $\left(\mathrm{s}_{\mathrm{c}}\right)$ )

\begin{tabular}{|c|c|c|c|c|c|c|}
\hline Gruppe & $\begin{array}{c}\text { I } \\
\text { weibl. } \\
\mathbf{n}=\mathbf{4 0}\end{array}$ & $\begin{array}{c}\text { II } \\
\text { Kastr. } \\
\mathbf{n}=39\end{array}$ & $\begin{array}{c}\text { III } \\
\text { Eber } \\
\mathbf{n}=\mathbf{3 9}\end{array}$ & $\begin{array}{c}\text { IV } \\
\text { Eber } \\
\mathbf{n = 3 7}\end{array}$ & $\begin{array}{c}\mathbf{V} \\
\begin{array}{c}\text { Eber } \\
\mathbf{n}=35\end{array}\end{array}$ & $\begin{array}{c}\text { VI } \\
\text { Eber } \\
\mathbf{n}=37\end{array}$ \\
\hline $\begin{array}{l}\text { Gewicht } 180 \mathrm{Tg} . \\
\text { (Prüfende) }\end{array}$ & $\begin{array}{cc}103,8 & 2,06 \\
\text { a } & \end{array}$ & $\begin{array}{cc}109,4 & 2,09 \\
\mathrm{ab} & \end{array}$ & $\begin{array}{cc}105,1 & 2,09 \\
\text { a } & \end{array}$ & $\begin{array}{c}104,2 \quad 2,15 \\
\text { a }\end{array}$ & $\begin{array}{cc}111,8 & 2,21 \\
b & \end{array}$ & $\begin{array}{cc}109,2 & 2,15 \\
\mathrm{ab} & \end{array}$ \\
\hline $\begin{array}{l}\text { tägl.Zunahme im } \\
\text { Prüfzeitraum (g) }\end{array}$ & $\begin{array}{c}769,7 \quad 17,7 \\
\text { a }\end{array}$ & $\begin{array}{l}854,2 \\
\text { b }\end{array}$ & $\begin{array}{l}806,4 \quad 17,9 \\
\text { ac }\end{array}$ & $\begin{array}{l}801,1 \quad 18,4 \\
\text { ac }\end{array}$ & $\begin{array}{c}861,318,9 \\
\text { b }\end{array}$ & $\begin{array}{l}839,1 \quad 18,4 \\
\text { bc }\end{array}$ \\
\hline $\begin{array}{l}\text { Futter- } \\
\text { verwertung (1:) }\end{array}$ & $\begin{array}{l}2,640,03 \\
\text { a }\end{array}$ & $\begin{array}{l}2,650,04 \\
\text { a }\end{array}$ & $\begin{array}{l}2,440,04 \\
b\end{array}$ & $\begin{array}{l}2,440,04 \\
b\end{array}$ & $\begin{array}{l}2,340,04 \\
\text { c }\end{array}$ & $\begin{array}{l}2,380,04 \\
\text { bc }\end{array}$ \\
\hline
\end{tabular}

abc: unterschiedliche Buchstaben zwischen den Gruppen zeigen signifikante Differenzen an

gehalt im Futter sowie die Sauengruppe (Kontrolle) niedrigere Gewichte erreichten. In der Futterverwertung unterschieden sich die vier Ebergruppen signifikant von den beiden Kontrollen, wobei die beiden Ebergruppen mit etwas höheren Lysingaben die tendenziell günstigsten Ergebnisse erzielten.

Der Grund hierfür ist im wesentlichen in der geringeren Fettbildung der Eber zu suchen, wie aus der folgenden Tabelle 3 über den Schlachtkörperwert hervorgeht.

\section{Merkmale des Schlachtkörperwertes}

Die geringsten Speckdicken wurden bei den vier Ebergruppen festgestellt. Die etwas höheren Maße der Sauen waren nicht signifikant, während die Börge mit dem höchsten Durchschnittswert von 23,4 mm alle übrigen Gruppen signifikant übertrafen. Die subjektive Bauchbewertung wies für die Kastraten ebenfalls die ungünstigsten Zahlen auf. Die Rückenmuskelquerschnittsfläche unterschied sich zwischen den vier Ebergruppen und den Kastraten nicht signifikant, lediglich die Sauen hoben sich deutlich darüber hinaus. Dies führte schließlich trotz des höheren Speckdickenmaßes zu einem ähnlichen Fleisch:Fett-Verhältnis wie bei den Ebern der Gruppe V und VI, und war gleichzeitig signifikant besser als das Fleisch:Fett-Verhältnis der Börge und der Ebergruppen III und IV. Der größere Rückenmuskelquerschnitt der Sauen führte schließlich auch zu dem höchsten Fleischanteil, gemessen mit FOM, der sich signifikant von den übrigen Gruppen unterschied, die ihrerseits nicht signifikant verschieden voneinander waren. Diese Ergebnisse wurden auch durch die Anwendung der Bonner Formel bestätigt.

Bei der FOM-Formel muss allerdings einschränkend bemerkt werden, dass sie nicht für Eberschlachtkörper entwickelt wurde. Es ist daher möglich, dass der Fleischanteil der Eber unterschätzt wird (DOBROWOLSKI et al., 1995).

\section{Merkmale der Fleischbeschaffenheit}

Merkmale der Fleischbeschaffenheit sind in Tabelle 4 zusammengefaßt. Die Messungen erfolgten im M. longissimus dorsi. Die drei Merkmale $\mathrm{pH}_{1}$ (45 Min. p.m.), Leitfä- 
Tabelle 3

Merkmale des Schlachtkörperwertes (LSQ-Mittelwerte $(\mu+c)$ und Standardfehler $(\mathrm{sc})$ ) (Carcass composition (LSQ means $(\mu+c)$ and standard errors $\left.\left(s_{c}\right)\right)$ )

\begin{tabular}{|c|c|c|c|c|c|c|c|c|c|c|c|c|}
\hline \multirow{2}{*}{$\begin{array}{l}\text { Gruppe } \\
\begin{array}{l}\text { Schlachtkörper- } \\
\text { gewicht (kg) }\end{array}\end{array}$} & \multicolumn{2}{|c|}{$\begin{array}{c}\text { I } \\
\text { weibl. } \\
\mathbf{n}=40\end{array}$} & \multicolumn{2}{|c|}{$\begin{array}{c}\text { II } \\
\text { Kastr. } \\
\mathbf{n}=39\end{array}$} & \multicolumn{2}{|c|}{$\begin{array}{c}\text { III } \\
\text { Eber } \\
\mathrm{n}=39\end{array}$} & \multicolumn{2}{|c|}{$\begin{array}{c}\text { IV } \\
\text { Eber } \\
\mathbf{n}=37\end{array}$} & \multicolumn{2}{|c|}{$\begin{array}{c}\mathbf{V} \\
\text { Eber } \\
\mathbf{n}=35\end{array}$} & \multicolumn{2}{|c|}{$\begin{array}{c}\text { VI } \\
\text { Eber } \\
n=37\end{array}$} \\
\hline & $\begin{array}{c}82,9 \\
\text { a }\end{array}$ & 1,71 & $\begin{array}{c}86,4 \\
\text { a }\end{array}$ & 1,74 & $\begin{array}{c}82,4 \\
\text { a }\end{array}$ & 1,74 & $\begin{array}{c}80,1 \\
\text { a }\end{array}$ & 1,78 & $\begin{array}{c}86,5 \\
\text { a }\end{array}$ & 1,83 & $\begin{array}{c}84,6 \\
\text { a }\end{array}$ & 1,78 \\
\hline $\begin{array}{l}\text { Schíachtkörper- } \\
\text { länge }(\mathrm{cm})\end{array}$ & $\begin{array}{c}97,3 \\
\text { a }\end{array}$ & 0,45 & $\begin{array}{l}95,1 \\
\mathrm{bc}\end{array}$ & 0,45 & $\begin{array}{c}96,8 \\
\mathrm{a}\end{array}$ & 0,45 & $\begin{array}{c}98,5 \\
b\end{array}$ & 0,46 & $\begin{array}{l}97,1 \\
a b\end{array}$ & 0,48 & $\begin{array}{l}97,6 \\
a b\end{array}$ & 0,46 \\
\hline $\begin{array}{l}\text { Speckdicke (mm) } \\
\text { (Ø } 3 \text { Meßst.) }\end{array}$ & $\begin{array}{c}21,2 \\
a\end{array}$ & 0,36 & $\begin{array}{l}23,4 \\
b c\end{array}$ & 0,36 & $\begin{array}{c}20,2 \\
a\end{array}$ & 0,36 & $\begin{array}{l}19,6 \\
a b\end{array}$ & 0,37 & $\begin{array}{l}19,3 \\
a b\end{array}$ & 0,38 & $\begin{array}{l}18,7 \\
a b\end{array}$ & 0,37 \\
\hline $\begin{array}{l}\text { Bauchpunkte } \\
(9=\text { max. })^{1)}\end{array}$ & $\begin{array}{l}7,1 \\
\text { a }\end{array}$ & 0,16 & $\begin{array}{l}5,6 \\
b\end{array}$ & 0,16 & $\begin{array}{l}6,4 \\
\text { ac }\end{array}$ & 0,16 & $\begin{array}{l}6,3 \\
b\end{array}$ & 0,17 & $\begin{array}{l}6,6 \\
\text { ac }\end{array}$ & 0,17 & $\begin{array}{l}6,8 \\
a\end{array}$ & 0,17 \\
\hline $\begin{array}{l}\text { Fläche des } \\
\text { M.long.dorsi }\left(\mathrm{cm}^{2}\right)\end{array}$ & $\begin{array}{c}52,5 \\
\text { a }\end{array}$ & 0,62 & $\begin{array}{l}48,3 \\
b c\end{array}$ & 0,63 & $\begin{array}{l}46,6 \\
b c\end{array}$ & 0,63 & $\begin{array}{l}44,2 \\
b d\end{array}$ & 0,66 & $\begin{array}{l}48,8 \\
b c\end{array}$ & 0,67 & $\begin{array}{l}46,5 \\
b c\end{array}$ & 0,65 \\
\hline $\begin{array}{l}\text { Fleisch:Fett- } \\
\text { verhälinis (1:) }\end{array}$ & $\begin{array}{l}0,25 \\
\text { a }\end{array}$ & 0,009 & $\begin{array}{l}0,34 \\
b\end{array}$ & 0,009 & $\begin{array}{l}0,30 \\
\text { ac }\end{array}$ & 0,009 & $\begin{array}{l}0,31 \\
\mathrm{c}\end{array}$ & 0,010 & $\begin{array}{l}0,27 \\
\text { ac }\end{array}$ & 0,010 & $\begin{array}{l}0,27 \\
a\end{array}$ & 0,010 \\
\hline $\begin{array}{l}\text { Magerfleischan- } \\
\text { teil in \% (FOM) }\end{array}$ & $\begin{array}{c}57,2 \\
\text { a }\end{array}$ & 0,34 & $\begin{array}{c}54,2 \\
b\end{array}$ & 0,34 & $\begin{array}{l}55,0 \\
b c\end{array}$ & 0,34 & $\begin{array}{l}54,2 \\
\mathrm{~b}\end{array}$ & 0,35 & $\begin{array}{l}55,5 \\
\mathrm{bc}\end{array}$ & 0,36 & $\begin{array}{l}55,0 \\
\mathrm{~b}\end{array}$ & 0,35 \\
\hline $\begin{array}{l}\text { Magerfleischan- } \\
\text { teil in \% (Bonn) }\end{array}$ & $\begin{array}{c}60,8 \\
\mathrm{a}\end{array}$ & 0,27 & $\begin{array}{c}57,9 \\
b\end{array}$ & 0,27 & $\begin{array}{l}58,6 \\
\text { bc }\end{array}$ & 0,27 & $\begin{array}{c}58,0 \\
b\end{array}$ & 0,28 & $\begin{array}{l}59,8 \\
b c\end{array}$ & 0,28 & $\begin{array}{l}59,3 \\
\text { bc }\end{array}$ & 0,28 \\
\hline
\end{tabular}

abe: unterschiedliche Buchstaben zwischen den Gruppen zeigen signifikante Differenzen an; "subjekt. Bewertungssystem bis 1997 goltig

higkeit ${ }_{24}$ (24 Std. p.m.) und Farbhelligkeit (24 Std. p.m.) wiesen zwischen den Gruppen nur geringe Unterschiede auf. Lediglich in der Leitfähigkeit ergaben sich für die Gruppe III etwas höhere und damit ungünstigere Werte; dies steht in guter Übereinstimmung mit dem relativ niedrigen - aber nicht signifikant verschiedenen - $\mathrm{pH}_{1}$-Wert. Der intramuskuläre Fettgehalt lag in allen Gruppen unter dem erwünschten Mindestwert von $2,0 \%$. Für den überraschend hohen Wert von 1,72\% in der Gruppe IV gibt es keine zufriedenstellende Erklärung; eher wäre bei den Kastraten mit der höheren Verfettung ein hoher Meßwert zu erwarten gewesen. Dies trifft mit 1,52\% auch zu, wenn von der Gruppe IV abgesehen wird. Hier ist allerdings anzumerken, daß über die Vatertiere nur bekannt war, daß sie der Rasse Piétrain angehörten, die aber bekanntermaBen in ihren Linien durchaus unterschiedliche Fleischbeschaffenheiten liefern können.

\section{Ebergeruch}

In Verbindung mit den anabol wirkenden männlichen Steroidhormonen tritt das An drostenon auf, ein Pheromon, das keine unmittelbare Hormonwirkung hat, aber leicht fettlöslich ist und den unangenehmen urinartigen Ebergeruch bei Erwärmen des Fettes hervorruft (CLAUS, 1991). In der vorliegenden Untersuchung wurde nicht das Andro- 
Tabelle 4

Merkmale der Fleischbeschaffenheit (LSQ-Mittelwerte $(\mu+c)$ und Standardfehler $\left(s_{c}\right)$ ) (Meat quality characteristics (LSQ means $(\mu+c)$ and standard errors $\left.\left(s_{c}\right)\right)$ )

\begin{tabular}{|c|c|c|c|c|c|c|c|c|c|c|c|c|}
\hline \multirow{2}{*}{$\begin{array}{l}\text { Gruppe } \\
\mathrm{pH}_{1} \text { (M.l.d.) }\end{array}$} & \multicolumn{2}{|c|}{$\begin{array}{c}\mathrm{I} \\
\text { weibl. } \\
\mathrm{n}=40\end{array}$} & \multicolumn{2}{|c|}{$\begin{array}{c}\text { II } \\
\text { Kastr. } \\
\mathbf{n}=39\end{array}$} & \multicolumn{2}{|c|}{$\begin{array}{c}\text { III } \\
\text { Eber } \\
\mathbf{n}=39\end{array}$} & \multicolumn{2}{|c|}{$\begin{array}{c}\text { IV } \\
\text { Eber } \\
\mathbf{n}=37\end{array}$} & \multicolumn{2}{|c|}{$\begin{array}{c}\text { V } \\
\text { Eber } \\
\mathbf{n}=35\end{array}$} & \multicolumn{2}{|c|}{$\begin{array}{c}\text { VI } \\
\text { Eber } \\
\mathbf{n}=37\end{array}$} \\
\hline & $\begin{array}{c}5,94 \\
\text { a }\end{array}$ & 0,05 & $\begin{array}{c}5,98 \\
\text { a }\end{array}$ & 0,05 & $\begin{array}{c}5,86 \\
\text { a }\end{array}$ & 0,05 & $\begin{array}{c}5,88 \\
\text { a }\end{array}$ & 0,05 & $\begin{array}{c}5,93 \\
\text { a }\end{array}$ & 0,05 & $\begin{array}{c}5,99 \\
a\end{array}$ & 0,05 \\
\hline $\begin{array}{l}\text { Leitfähigkeit } 24 \\
(\mathrm{mS})\end{array}$ & $\begin{array}{c}7,44 \\
\text { a }\end{array}$ & 0,57 & $\begin{array}{c}6,07 \\
\text { a }\end{array}$ & 0,58 & $\begin{array}{c}8,03 \\
\text { ac }\end{array}$ & 0,58 & $\begin{array}{c}7,65 \\
\text { a }\end{array}$ & 0,60 & $\begin{array}{c}5,94 \\
a b\end{array}$ & 0,62 & $\begin{array}{c}5,67 \\
b\end{array}$ & 0,60 \\
\hline $\begin{array}{l}\text { Farbhelligkeit } \\
\text { (Minolta L) }\end{array}$ & $\begin{array}{c}48,25 \\
\text { a }\end{array}$ & 0,37 & $\begin{array}{c}48,09 \\
\mathrm{ab}\end{array}$ & 0,37 & $\begin{array}{c}48,45 \\
\text { a }\end{array}$ & 0,37 & $\begin{array}{c}47,67 \\
\text { a }\end{array}$ & 0,39 & $\begin{array}{c}47,93 \\
\text { a }\end{array}$ & 0,40 & $\begin{array}{c}47,11 \\
b\end{array}$ & 0,38 \\
\hline $\begin{array}{l}\text { Fettgehalt (NIT) } \\
\text { in } \%\end{array}$ & $\begin{array}{c}1,19 \\
\text { a }\end{array}$ & 0,06 & $\begin{array}{c}1,52 \\
b c\end{array}$ & 0,06 & $\begin{array}{l}1,27 \\
b c\end{array}$ & 0,06 & $\begin{array}{l}1,72 \\
\text { bd }\end{array}$ & 0,06 & $\begin{array}{l}1,10 \\
b c\end{array}$ & 0,06 & $\begin{array}{l}1,26 \\
b c\end{array}$ & 0,06 \\
\hline $\begin{array}{l}\text { Wassergehalt } \\
\text { (NIT) in \% }\end{array}$ & $\begin{array}{r}74,5 \\
a\end{array}$ & 0,06 & $\begin{array}{r}74,2 \\
b\end{array}$ & 0,06 & $\begin{array}{r}74,8 \\
\mathrm{bc}\end{array}$ & 0,06 & $\begin{array}{r}74,7 \\
\mathrm{bc}\end{array}$ & 0,06 & $\begin{array}{r}74,8 \\
b c\end{array}$ & 0,06 & $\begin{array}{r}74,7 \\
b c\end{array}$ & 0,06 \\
\hline
\end{tabular}

abc: unterschiedliche Buchstaben zwischen den Gruppen zeigen signifikante Differenzen an

stenon selbst, sondern das eng damit korrelierte Testosteron analysiert. Dieser Weg wurde deshalb gewählt, weil Testosteron im Blut nachweisbar ist, während Androstenon überwiegend im Fett gelöst ist und deshalb die Blutwerte nicht repräsentativ wären. Die Analysenwerte sind in Tabelle 5 dargestellt und zeigen insbesondere im Bereich zwischen $60 \mathrm{~kg}$ und dem Mastende einen sehr deutlichen Anstieg in allen Ebergruppen, während sich bei den Kastraten der Wert nur unwesentlich erhöhte. Dieser Vorgang läßt erkennen, daß die Geschlechtsreife zum Zeitpunkt der Schlachtung bereits eingetreten war oder unmittelbar bevorstand. Die Ergebnisse der sensorischen Prüfung in der letzten Zeile der Tabelle 5 unterscheiden sich für alle vier Ebergruppen signifikant zu den beiden Kontrollen, $d$. h. den weiblichen und männlich kastrierten Tieren. Im Durchschnitt waren also alle Ebergruppen mit deutlichem Ebergeruch behaftet.

\section{Skatolgehalte im Fettgewebe}

Skatol und Indol entstehen durch den mikrobiellen Abbau der Aminosäure Tryptophan, und insbesondere das Skatol verursacht einen fäkalartigen Geruch oder Geschmack (WEILER et al., 1995). Als Grenzwert werden 240 ng Skatol/g Fett genannt, d. h. ab dieser Konzentration ist die Geruchs- bzw. Geschmacksabweichung deutlich wahrnehmbar. In der vorliegenden Untersuchung zeigen die Zahlen der Tabelle 6, daß im Durchschnitt die Meßwerte für alle vier Ebergruppen deutlich über denen der Kontrollen lagen. Die Gruppe VI unterschied sich allerdings nicht signifikant von der weiblichen Kontrollgruppe. Die großen Variationen innerhalb der Gruppen, die auch in den Extremwerten zum Ausdruck kommen, zeigen aber einen weiten Überschneidungsbereich zwischen den Geschlechtern. Der genannte kritische Wert wurde aller- 
Arch. Ticrz. 42 (1999) 6

dings nur von wenigen Ebern erreicht, weibliche Tiere und Börge blieben in allen Fällen darunter.

Tabelle 5

Plasma-Testosteron- und Progesteronwerte (in ng/ml) (LSQ-Mittelwerte $(\mu+\mathrm{c}$ ) und Standardfehler (sc)) (Plasma-Testosterone and Progesterone values (in ng/ml) ((LSQ means $(\mu+\mathrm{c})$ and standard errors $\left.\left(\mathrm{s}_{\mathrm{c}}\right)\right)$

\begin{tabular}{|c|c|c|c|c|c|c|c|c|c|c|c|c|}
\hline \multirow{2}{*}{$\begin{array}{l}\text { Gruppe } \\
\text { Tesosteron } \\
30 \mathrm{~kg}\end{array}$} & \multicolumn{2}{|c|}{$\begin{array}{c}\text { I } \\
\text { weibl. }\end{array}$} & \multicolumn{2}{|c|}{$\begin{array}{c}\text { II } \\
\text { Kastr. }\end{array}$} & \multicolumn{2}{|c|}{$\begin{array}{c}\text { III } \\
\text { Eber }\end{array}$} & \multicolumn{2}{|c|}{$\begin{array}{l}\text { IV } \\
\text { Eber }\end{array}$} & \multicolumn{2}{|c|}{$\begin{array}{c}\text { V } \\
\text { Eber }\end{array}$} & \multicolumn{2}{|c|}{$\begin{array}{c}\text { VI } \\
\text { Eber }\end{array}$} \\
\hline & - & & $\begin{array}{c}0,19 \\
\text { a }\end{array}$ & 0,18 & $\begin{array}{c}1,07 \\
b\end{array}$ & 0,18 & $\begin{array}{c}0,92 \\
b\end{array}$ & 0,19 & $\begin{array}{c}1,79 \\
\mathrm{c}\end{array}$ & 0,20 & $\begin{array}{c}1,44 \\
\mathrm{c}\end{array}$ & 0,18 \\
\hline $\begin{array}{l}\text { Testosteron } \\
60 \mathrm{~kg}\end{array}$ & -. & & $\begin{array}{c}0,15 \\
\text { a }\end{array}$ & 0,43 & $\begin{array}{c}2,51 \\
b\end{array}$ & 0,42 & $\begin{array}{c}1,52 \\
b\end{array}$ & 0,43 & $\begin{array}{c}2,84 \\
\mathrm{c}\end{array}$ & 0,43 & $\begin{array}{c}3,12 \\
\mathrm{c}\end{array}$ & 0,43 \\
\hline $\begin{array}{l}\text { Testosteron } \\
\text { (Schlachtblut) }\end{array}$ & - & & $\begin{array}{c}0,30 \\
\text { a }\end{array}$ & 1,88 & $\begin{array}{r}11,0 \\
b\end{array}$ & 1,80 & $\begin{array}{r}15,6 \\
b\end{array}$ & 1,88 & $\begin{array}{r}17,7 \\
\mathrm{c}\end{array}$ & 1,88 & $\begin{array}{r}17,7 \\
\mathrm{c}\end{array}$ & 1,82 \\
\hline $\begin{array}{l}\text { Progesteron } \\
\text { (Minolta L) }\end{array}$ & 1,31 & 1,79 & - & & - & & - & & - & & - & \\
\hline $\begin{array}{l}\text { Sensorik } \\
\text { (1 bis } 4 \text { Pkt.) }\end{array}$ & $\begin{array}{c}1,89 \\
\text { a }\end{array}$ & 0,07 & $\begin{array}{c}1,93 \\
\text { a }\end{array}$ & 0,07 & $\begin{array}{c}2,37 \\
\mathrm{~b}\end{array}$ & 0,07 & $\begin{array}{c}2,27 \\
b\end{array}$ & 0,08 & $\begin{array}{c}2,42 \\
\mathrm{~b}\end{array}$ & 0,08 & $\begin{array}{c}2,22 \\
b\end{array}$ & 0,08 \\
\hline
\end{tabular}

abc: unterschiedliche Buchstaben zwischen den Gruppen zeigen signifikante Differenzen an

Tabelle 6

Skatolgehalt in Fettproben (Flomen) in ng/g (Skatole content in fat samples (kidney fat) in ng/g)

\begin{tabular}{l|cccccc}
\hline Gruppe & $\begin{array}{c}\text { I } \\
\text { Kontrolle }(\mathrm{w})\end{array}$ & $\begin{array}{c}\text { II } \\
\text { Kontrolle } \\
(\mathrm{Bö})\end{array}$ & $\begin{array}{c}\text { Eber } \\
9 / 160\end{array}$ & $\begin{array}{c}\text { Eber } \\
9 / 180\end{array}$ & $\begin{array}{c}\text { Eber } \\
9 / 180\end{array}$ & $\begin{array}{c}\text { Eber } \\
10 / 180\end{array}$ \\
\hline $\mathrm{n}$ & 37 & 39 & 36 & 35 & 32 & 35 \\
$\mathrm{H}+\mathrm{c}$ & $43,3 \mathrm{a}$ & $35,2 \mathbf{b}$ & $\mathbf{7 5 , 8}$ a & $\mathbf{7 1 , 5}$ a & $\mathbf{7 5 , 9}$ a & $\mathbf{6 2 , 3}$ ab \\
$\mathrm{S}_{\mathrm{c}}$ & 33,7 & 20,0 & 58,8 & 50,3 & 55,4 & 42,9 \\
$\min$ & 4,3 & 2,2 & 14,3 & 14,7 & 17,6 & 11,5 \\
$\max$. & 137,7 & 75,3 & 212,5 & 263,2 & 254,6 & 203,8 \\
\hline
\end{tabular}

abe: unterschiedliche Buchstaben zwischen den Gruppen zeigen signifikante Differenzen an

\section{Diskussion und Schlussfolgerungen}

Die Untersuchungsergebnisse bestätigen die Überlegenheit der Ebermast nicht in allen Punkten, wie es mehrfach in der Literatur beschrieben wurde. Das Muskelwachstum pro Zeiteinheit unterschied sich zwischen den Gruppen und den Geschlechtern nur unwesentlich. Die Fettbildung dagegen war in allen Ebergruppen im Vergleich zu den männlichen Kastraten reduziert, in der Tendenz auch gegenüber den weiblichen Tieren. Dieses Ergebnis deutet darauf hin, daß das Fleischbildungsvermögen durch die 
ständige Selektion auf dieses Leistungsmerkmal in allen Kategorien stark ausgeprägt ist, so daß daraus kein wesentlicher Vorteil der Ebermast entsteht. Die reduzierte Fettbildung führt allerdings zu einer Verbesserung der Futterverwertung.

Die Nährstoffkonzentration in den angebotenen Futtermischungen reichte offensichtlich sowohl vom Energiegehalt als auch vom Eiweiß- und Lysinanteil aus, um das hohe genetisch verankerte Muskelbildungsvermögen auszuschöpfen. Die Zulage bei beiden Eiweißkomponenten führte zu keiner nennenswerten Leistungsverbesserung. Lediglich die Futterverwertung in den beiden Ebergruppen mit den höchsten Eiweißanteilen im Futter konnte geringfügig verbessert werden.

Die Fleischbeschaffenheit unterschied sich bei hohem Niveau zwischen den Gruppen nicht. Hierin zeigt sich die muskelphysiologische Stabilität und Ausgeglichenheit systematischer Kreuzungsprodukte. Da auch keine wesentlichen Unterschiede in der geweblichen Körperzusammensetzung bestanden, konnte der "Merkmalsantagonismus" nicht zur Wirkung gelangen.

Die im Versuchszeitraum erzielten Mastendgewichte zwischen 104 und $112 \mathrm{~kg}$ Lebendgewicht führten zu Schlachtkörpern im Gewichtsbereich zwischen 80 und $86 \mathrm{~kg}$, also einer Größenordnung, die gerade oberhalb der durch die EWG-Richtlinie gesetzten Grenze liegt. Daß dennoch alle Ebergruppen im Durchschnitt deutliche Geruchsabweichungen aufwiesen, läßt erkennen, daß diese Grenzziehung zu hoch gegriffen ist. Demnach ist bei einem Schlachtgewicht von bis zu $80 \mathrm{~kg}$ in zahlreichen Fällen mit dem Auftreten von Ebergeruch zu rechnen. Das Skatol als Indikator für das Vorliegen von Ebergeruch ist nach den Ergebnissen dieser Untersuchung nicht geeignet. Die Öberschneidungen der Gehaltszahlen im Fett (Flomen) lassen erkennen, daß auch weibliche Tiere und Kastraten nicht frei von Skatol sind. Sie erreichten allerdings nach den vorliegenden Ergebnissen nicht die kritische Grenze von $250 \mathrm{ng} / \mathrm{g}$.

Die Freistellung von einer diesbezüglichen Prüfung ist also sachlich nicht gerechtfertigt. Unbeabsichtigt oder ungeprüft in den Verkehr gebrachte Schlachthälften mit Ebergeruch können zu einem Imageverlust von Schweinefleisch beim Verbraucher führen, weil er die Ursache für das Auftreten unangenehmer Geschmacks- und Geruchsabweichungen im Sinne von Eber- und Skatolgeruch nicht kennt. Dies trifft aber offensichtlich nicht für alle Länder der EU zu.

Die nur unwesentlichen wirtschaftlichen Vorteile der Ebermast und das erhebliche Risiko des Auftretens von Ebergeruch haben zu einen deutlichen Rückgang der Ebermast in den europäischen Ländern geführt, soweit es sich um Schlachtgewichte von über 80 $\mathrm{kg}$ handelt. Anders verhält es sich dagegen bei der Erzeugung leichterer Schlachtkörper im Zusammenhang mit der Bacon-Produktion.

\section{Danksagung}

Die Autoren danken den Mitarbeitern der Landwirtschaftskammer Hannover, den Herren Dr. Röver ( $\dagger$ ) und Schön, für ihre tatkräftige Unterstützung bei der Durchführung der Untersuchung sowie Herrn Prof. Elsaesser (TZV Mariensee) für die Hormonanalysen. 
CLAUS, R.:

\section{Literatur}

Ebermast - eine Wertung aus physiologischer Sicht. VET 10 (1991), 6-14

DOBROWOLSKI, A.; HÖRETH, R.; BRANSCHEID, W.:

Der Schlachtkörperwert von Ebern und Börgen und Probleme der Klassifizierung. Angewandte Wissenschaft 449 (1995), 6-13

GIBBS, M.; DEHNHARD, M.; FISCHER, A.:

Bestimmung von Skatol und Indol in Rückenspeck und Muskelfleisch von Schweinen durch Hochleistungs-Flüssigkeitschromatographie (HPLC) mit fluorimetrischer Detektion. Z. Lebensm. Unters. Forsch 193 (1991), 220-223

WEILER, U.; DEHNHARD, M.; HERBERT, E.; CLAUS, R.:

Einfluss von Geschlecht, Genotyp und Mastendgewicht auf die Androstenon- und Skatolkonzentrationen im Fett von Mastschweinen. Angewandte Wissenschaft 449 (1995), 14-32

Eingegangen:17.09.1999

Akzepticrt: 12.10 .1999

Anschriften der Verfasser

Prof. Dr. ERHARD KALLWEIT, Prof. Dr. NAHID PARVIZI, Dr. FRANC KLOBASA,

Dr. MARTINA HENNING

Institut für Tierzucht und Tierverhalten Neustadt-Mariensee

Höltystrasse 10

D-31535 Neustadt

Dr. HARTWIG BÖHME

Institut für Tierernährung der Bundesforschungsanstalt

für Landwirtschaft Braunschweig-Völkenrode

Bundesallee 50

D-38116 Braunschweig 
Arch. Tierz., Dummerstorf 42 (1999) 6, 592

\title{
Buchbesprechung
}

\author{
Fertilitätsstörungen beim weiblichen Rind \\ EBERHARD GRUNERT und AART DE KRUIF
}

3. neubearbeitete Auflage, 430 Seiten, 446 Abbildungen, davon 130 auf Farbtafeln, 49 Tabellen, Parey Buchverlag Berlin, 1999, ISBN 3-8263-3150-8, 248,00 DM

Die Monographie Fertilitätsstörungen beim weiblichen Rind zählt seit ihrer ersten Auflage zu den deutschsprachigen Standardwerken auf dem Fachgebiet. Unter Mitarbeit nationaler und internationaler Autoren haben die beiden Herausgeber Eberhard Grunert und Aart de Kruif in der nunmehr dritten Auflage des Lehrbuches das aktuelle Wissen auf dem Gebiet in 21 Kapiteln strukturiert. Es erscheint bereits einleitend bemerkenswert, daß es den Herausgebern gelungen ist, den Umfang des Lehrbuches im Vergleich zur zweiten Auflage zu reduzieren, obwohl zahlreiche neue Erkenntnisse in die einzelnen Abschnitte eingearbeitet wurden. Die Vereinigung beider Zielstellungen wurde nicht nur durch einen Verzicht auf die in der ersten und zweiten Auflage vorkommenden Kapitel „Bedeutung der Fruchtbarkeitsstörungen“ und „Embryologie und Anatomie des weiblichen Genitale“, sondern vor allem durch eine konsequente Überarbeitung des gesamten Lehrbuches ermöglicht. Als Beispiele für die Integration von neuen Erkenntnissen in die dritte Auflage seien die Ausführungen zur Anwendung der bildgebenden Ultraschalldiagnostik im Rahmen der Trächtigkeitsdiagnostik oder die Informationen über neuere Reproduktionstechniken genannt. Den Autoren der einzelnen Abschnitte ist es in hervorzuhebender Weise gelungen, dem Leserkreis die wesentlichsten Informationen zu den verschiedenen Teilthemen in kurzer, übersichtlicher und einprägsamer Form zu vermitteln. Einen wesentlichen Beitrag dazu leisten die informativen Schemata, die zahlreichen aussagefähigen und häufig auch farbigen Abbildungen sowie die übersichtlichen Tabellen. Das prinzipielle didaktische Konzept des Lehrbuches, welches von physiologischen Grundlagen ausgehend über eine fundierte Diagnostik die gezielte und effektive Therapie bzw. Prophylaxe von Fortpflanzungsstörungen zum Ziel hat, wurde sowohl durch die Gliederung der Thematik unter inhaltlichen Aspekten als auch durch eine ausgewogene Verbindung von Text und anderen gestalterischen Elementen sehr gut umgesetzt. In diesem $\mathrm{Zu}$ sammenhang wird auf das Bestreben der Autoren verwiesen, den Lesern bei allen Bemühungen um inhaltliche Aktualität der einzelnen Kapitel gesicherte Erkenntnisse zu vermitteln, die eine praktische Anwendung dieser gestatten. Gerade in der Verbindung von Wissensvermittlung und der Nutzung von Erkenntnissen in der tierärztlichen Praxis liegt der besondere Wert des Lehrbuches, welches dem interessierten Leserkreis in seiner neuesten Auflage bestens empfohlen werden kann. 